\title{
On a Family of Hypergeometric Sobolev Orthogonal Polynomials on the Unit Circle
}

\author{
SERGEY M. ZAGORODNYUK*
}

ABSTRACT. In this paper, we study the following family of hypergeometric polynomials:

$y_{n}(x)=\frac{(-1)^{\rho}}{n !} x^{n}{ }_{2} F_{0}\left(-n, \rho ;-;-\frac{1}{x}\right)$, depending on a parameter $\rho \in \mathbb{N}$. Differential equations of orders $\rho+1$ and 2 for these polynomials are given. A recurrence relation for $y_{n}$ is derived as well. Polynomials $y_{n}$ are Sobolev orthogonal polynomials on the unit circle with an explicit matrix measure.

Keywords: Sobolev orthogonal polynomials, hypergeometric polynomials, unit circle, differential equation, recurrence relation.

2010 Mathematics Subject Classification: 33C45, $42 \mathrm{C} 05$.

\section{INTRODUCTION}

The theories of orthogonal polynomials (OP) on the real line and on the unit circle have many similarities as well as considerable differences [19, 6, 16, 17]. For a long time, they have been developed side by side by efforts of numerous mathematicians. The theory of Sobolev orthogonal polynomials is a much more terra incognita $[11,18,10,8]$. In this theory, one can also see that some ideas come from the real line to the unit circle. Examples of such ideas are adding of Dirac deltas to the classical inner products and considering of coherent pairs of measures (see, e.g., [3,4] and references therein). In the present paper, we shall follow the same line: we shall develop the ideas from [20] to get some new hypergeometric polynomials and study their properties.

Let $\mu$ be a probability measure on $\mathbb{T}$ with an infinite support. We assume that $\mu$ is defined on a $\sigma$-algebra $\mathfrak{A}$ which contains $\mathfrak{B}(\mathbb{T})$. Denote by $p_{n}$ orthogonal polynomials on the unit circle (OPUC) with respect to $\mu\left(\operatorname{deg} p_{n}=n\right.$, but the positivity of leading coefficients is not assumed):

$$
\int_{\mathbb{T}} p_{n}(z) \overline{p_{m}(z)} d \mu=A_{n} \delta_{n, m}, \quad A_{n}>0, n, m \in \mathbb{Z}_{+} .
$$

Fix an arbitrary positive integer $\rho$. Consider the following differential equation:

$$
\left(e^{-x} y(x)\right)^{(\rho)}=e^{-x} p_{n}(x), \quad n \in \mathbb{Z}_{+} .
$$

Expanding the derivative by the Leibniz formula and canceling $e^{-x}$, we get:

$$
\sum_{k=0}^{\rho}(-1)^{\rho-k}\left(\begin{array}{c}
\rho \\
k
\end{array}\right) y^{(k)}(x)=p_{n}(x), \quad n \in \mathbb{Z}_{+} .
$$

Received: 17.02.2020; Accepted: 10.04.2020; Published Online: 11.04.2020

*Corresponding author: Sergey M. Zagorodnyuk; Sergey.M.Zagorodnyuk@gmail.com

DOI: $10.33205 / \mathrm{cma} .690236$ 
Condition A. Suppose that for each $n \in \mathbb{Z}_{+}$, there exists a $n$-th degree polynomial solution $y=y_{n}(x)$ of (1.3).

If Condition A is satisfied, then $y_{n}$ are Sobolev orthogonal polynomials on the unit circle (SOPUC):

$$
\int_{\mathbb{T}}\left(y_{n}(z), y_{n}^{\prime}(z), \ldots, y_{n}^{(\rho)}(z)\right) M \overline{\left(\begin{array}{c}
y_{m}(z) \\
y_{m}^{\prime}(z) \\
\vdots \\
y_{m}^{(\rho)}(z)
\end{array}\right)} d \mu=A_{n} \delta_{n, m}, \quad n, m \in \mathbb{Z}_{+},
$$

where

$$
M=\left((-1)^{l+j}\left(\begin{array}{c}
\rho \\
l
\end{array}\right)\left(\begin{array}{c}
\rho \\
j
\end{array}\right)\right)_{l, j=0}^{\rho} .
$$

Recall that the classical orthogonal polynomials $\left\{p_{n}(x)\right\}_{n=0}^{\infty}$ on $\mathbb{R}$ (namely, one of the following systems: Jacobi OP, Laguerre OP, Hermite OP) are eigenfunctions of a second-order linear differential operator $L$ (see, e.g., [9]):

$$
L p_{n}(x)=\lambda_{n} p_{n}(x), \quad n=0,1,2, \ldots .
$$

On the other hand, the vector $\vec{p}(x)=\left(p_{0}(x), p_{1}(x), \ldots\right)^{T}$ is an eigenvector of the corresponding Jacobi matrix $J$ :

$$
J \vec{p}(x)=x \vec{p}(x) .
$$

In the case of OPUC, we can not give any property of form (1.6) with some linear differential operator. In this case and, more generally, in the case of SOPUC the notion of operator pencils seems to be appropriate. Operator pencils appeared in the theory of biorthogonal rational functions, see [7, 21].

By operator pencils or operator polynomials one means polynomials of complex variable $\lambda$ whose coefficients are linear bounded operators acting in a Banach space $X([15,12])$ :

$$
L(\lambda)=\sum_{j=0}^{m} \lambda^{j} A_{j},
$$

where $A_{j}: X \rightarrow X(j=0, \ldots, m)$. In the case $m=1(m=2)$, the pencil is called linear (respectively quadratic). Operator pencils with differential operators $A_{j}$ appear in many physical problems, see ([13]) and references therein.

The following problem seems to be a suitable framework to study classical type SOPUC.

Problem 1. To describe all SOPUC $\left\{y_{n}(z)\right\}_{n=0}^{\infty}$, satisfying the following two properties:

(a) Polynomials $y_{n}(z)$ satisfy the following differential equation:

$$
R y_{n}(z)=\lambda_{n} S y_{n}(z), \quad n=0,1,2, \ldots,
$$

where $R, S$ are linear differential operators of finite orders, having polynomial coefficients not depending on $n ; \lambda_{n} \in \mathbb{C}$;

(b) Polynomials $y_{n}(z)$ satisfy the following difference equation:

$$
L \vec{y}(z)=z M \vec{y}(z), \quad \vec{y}(z)=\left(y_{0}(z), y_{1}(z), \ldots\right)^{T},
$$

where $L, M$ are semi-infinite complex banded (i.e. having a finite number of non-zero diagonals) matrices. 
Relation (1.9) means that $y_{n}(z)$ are eigenvalues of the operator pencil $R-\lambda S$, while relation (1.10) shows that vectors of $y_{n}(z)$ are eigenvalues of the operator pencil $L-z M$. For example, consider the following case: $y_{n}(x)=z^{n}$. They satisfy the differential equation:

$$
z\left(z^{n}\right)^{\prime}=n z^{n}, \quad n \in \mathbb{Z}_{+},
$$

and they obey (1.10) with $L$ being the identity semi-infinite matrix, $M$ being the semi-infinite matrix with all 1 on the first subdiagonal and 0 on other places.

Let us briefly describe the content of the paper. At first, we consider the following case: $p_{n}(x)=x^{n}$, and $\mu$ being the normalized arc length measure on $\mathbb{T}$. Equating coefficients of the same powers on the both sides of equation (1.3) one obtains a linear system of equations for the coefficients of an unknown polynomial $y(x)$ (the same idea was used in [2]). However, for large values of $\rho$ it is not easy to get a convenient expression for solutions, without huge determinants or recurrences. In this case, equation (1.2) turned out to be useful. It gives a possibility to express $y_{n}(x)$ for $\rho=1$ in terms of the incomplete gamma function. A step-by-step analysis for $\rho=1,2, \ldots$, allows to obtain an explicit representation of $y_{n}(x)$. Explicit representations, differential equations and orthogonality relations for $y_{n}$ will be given by Theorem 2.1. As a corollary, we obtain a solution to (1.3) in the general case (Corollary 2.1). Using Fasenmeier's method ([14]) for the reversed polynomials $y_{n}^{*}(x)=x^{n} y_{n}\left(\frac{1}{x}\right)$, we shall derive recurrence relations for $y_{n}(x)$ (Theorem 2.2) as well. Thus, we provide an example of SOPUC which satisfies conditions of Problem 1.

Notations. As usual, we denote by $\mathbb{R}, \mathbb{C}, \mathbb{N}, \mathbb{Z}, \mathbb{Z}_{+}$, the sets of real numbers, complex numbers, positive integers, integers and non-negative integers, respectively. The superscript $T$ means the transpose of a (finite or infinite) vector. Set $\mathbb{T}:=\{z \in \mathbb{C}:|z|=1\}$. By $\mathfrak{B}(\mathbb{T})$, we mean the set of all Borel subsets of $\mathbb{T}$. By $\mathbb{P}$, we denote the set of all polynomials with complex coefficients. For a complex number $c$, we denote $(c)_{0}=1,(c)_{k}=c(c+1) \ldots(c+k-1), k \in \mathbb{N}$ (the shifted factorial or Pochhammer symbol). The generalized hypergeometric function is denoted by

$$
{ }_{m} F_{n}\left(a_{1}, \ldots, a_{m} ; b_{1}, \ldots, b_{n} ; x\right)=\sum_{k=0}^{\infty} \frac{\left(a_{1}\right)_{k} \ldots\left(a_{m}\right)_{k}}{\left(b_{1}\right)_{k} \ldots\left(b_{n}\right)_{k}} \frac{x^{k}}{k !},
$$

where $m, n \in \mathbb{N}, a_{j}, b_{l} \in \mathbb{C}$.

\section{SOME SOBOLEV ORTHOGONAL POLYNOMIALS ON $\mathbb{T}$}

As it was stated in the Introduction, in what follows we shall consider the following case: $p_{n}(x)=x^{n}$, and $\mu=\mu_{0}$ being the (probability) normalized arc length measure on $\mathbb{T}$, which may be identified with the Lebesgue measure on $[0,2 \pi)$. Rewrite equations (1.2), (1.3) for this case:

$$
\begin{gathered}
\left(e^{-x} y_{n}(x)\right)^{(\rho)}=e^{-x} x^{n}, \quad n \in \mathbb{Z}_{+} ; \\
\sum_{k=0}^{\rho}(-1)^{\rho-k}\left(\begin{array}{c}
\rho \\
k
\end{array}\right) y_{n}^{(k)}(x)=x^{n}, \quad n \in \mathbb{Z}_{+} \cdot
\end{gathered}
$$

We start with the case $\rho=1$. In this case, equation (2.12) has the following form:

$$
y_{n}^{\prime}(x)-y_{n}(x)=x^{n}, \quad n \in \mathbb{Z}_{+} .
$$

Fix an arbitrary $n \in \mathbb{Z}_{+}$. We shall seek for a solution of the required form:

$$
y_{n}(x)=\sum_{k=0}^{n} \mu_{n, k} x^{k}, \quad \mu_{n, k} \in \mathbb{C} .
$$


Substitute for $y_{n}$ into (2.13) to get

$$
\sum_{k=0}^{n-1}\left\{(k+1) \mu_{n, k+1}-\mu_{n, k}\right\} x^{k}-\mu_{n, n} x^{n}=x^{n} .
$$

Comparing the coefficients of the same powers on the both sides, we obtain that

$$
\mu_{n, n}=-1, \quad \mu_{n, k}=(k+1) \mu_{n, k+1}, \quad k=n-1, n-2, \ldots, 0 .
$$

It can be verified by the induction argument that

$$
\mu_{n, j}=(-n)_{n-j}(-1)^{n-j-1}=-\frac{n !}{j !}, \quad j=0,1, \ldots, n .
$$

Thus,

$$
y_{n}(x)=-n ! \sum_{k=0}^{n} \frac{x^{k}}{k !}
$$

is a solution of (2.13). In the case $\rho>1$, it is not easy to solve the corresponding recurrence relation for the coefficients and we shall proceed in another way.

Observe that

$$
y_{n}(x)=-e^{x} \Gamma(n+1, x),
$$

where

$$
\Gamma(\alpha, x)=\int_{x}^{\infty} e^{-t} t^{\alpha-1} d t, \quad \alpha>0,
$$

is the complementary incomplete gamma function ([1]). In fact, integrating (2.11) (with $\rho=1$ ) from $a$ to $b(a, b \in \mathbb{R})$, we get

$$
e^{-b} y_{n}(b)-e^{-a} y_{n}(a)=\int_{a}^{b} e^{-x} x^{n} d x
$$

Taking limit when $b \rightarrow+\infty$, we get

$$
y_{n}(a)=-e^{a} \int_{a}^{\infty} e^{-x} x^{n} d x,
$$

and relation (2.17) follows.

Suppose that we have constructed a polynomial solution (of the required form) $y_{n}(\rho ; x)=$ $y_{n}(x)$ of equation (2.11) for some positive integer $\rho$. Let us show how to get a polynomial solution $y_{n}(\rho+1 ; x)$ of equation (2.11) with $\rho+1$. Notice that, we do not state the uniqueness of such solutions for $\rho>2$. We shall need the following auxiliary equation:

$$
\left(e^{-x} y_{n}(\rho+1 ; x)\right)^{\prime}=e^{-x} y_{n}(\rho ; x), \quad n \in \mathbb{Z}_{+},
$$

with an unknown $y_{n}(\rho+1 ; x)$. Equation (2.19) has a unique $n$-th degree polynomial solution. This can be verified comparing the coefficients of polynomials, in the same way as for equation (2.13). It is not easy to solve the corresponding recurrence relation in this case, but the existence and the uniqueness of a $n$-th degree polynomial solution is obvious.

Integrating relation (2.19) from $t$ to $b$, we get

$$
e^{-b} y_{n}(\rho+1 ; b)-e^{-t} y_{n}(\rho+1 ; t)=\int_{t}^{b} e^{-x} y_{n}(\rho ; x) d x
$$

Taking limit when $b \rightarrow+\infty$, we get

$$
y_{n}(\rho+1 ; t)=-e^{t} \int_{t}^{\infty} e^{-x} y_{n}(\rho ; x) d x .
$$


By (2.11), (2.19) we may write:

$$
e^{-x} x^{n}=\left(e^{-x} y_{n}(\rho ; x)\right)^{(\rho)}=\left(e^{-x} y_{n}(\rho+1 ; x)\right)^{(\rho+1)} .
$$

Therefore, $y_{n}(\rho+1 ; \cdot)$ given by (2.20) is a required polynomial solution of $(2.11)$ for $\rho+1$.

Equation (2.20) shows how to construct polynomial solutions step-by-step for $\rho=1,2, \ldots$ However, we are interested to get an explicit representation for every $y_{n}(\rho ; x)$. Let

$$
y_{n}(\rho ; x)=\sum_{j=0}^{n} d_{j}(\rho) \frac{x^{j}}{j !}, \quad n \in \mathbb{Z}_{+}, \rho \in \mathbb{N},
$$

with some unknown complex numbers $d_{j}(\rho)$. By (2.20), (2.18), (2.16) we may write

$$
\begin{aligned}
y_{n}(\rho+1 ; t) & =-e^{t} \sum_{j=0}^{n} d_{j}(\rho) \frac{1}{j !} \int_{t}^{\infty} e^{-x} x^{j} d x=\sum_{j=0}^{n} d_{j}(\rho) \frac{1}{j !} y_{j}(1 ; t) \\
& =-\sum_{j=0}^{n} \sum_{k=0}^{j} d_{j}(\rho) \frac{t^{k}}{k !}, \quad n \in \mathbb{Z}_{+}, \rho \in \mathbb{N} .
\end{aligned}
$$

Changing the order of summation in (2.22), we write:

$$
y_{n}(\rho+1 ; t)=-\sum_{k=0}^{n} \sum_{j=k}^{n} d_{j}(\rho) \frac{t^{k}}{k !} .
$$

Therefore,

$$
d_{k}(\rho+1)=-\sum_{j=k}^{n} d_{j}(\rho), \quad k=0,1, \ldots, n ; \rho \in \mathbb{N} .
$$

Relation (2.23) can be written in a matrix form for the vectors of coefficients $\vec{d}(\rho):=\left(d_{0}(\rho), \ldots, d_{n}(\rho)\right)^{T}$, and a $(n+1) \times(n+1)$ upper-diagonal Toeplitz matrix $T$, having all nonzero elements equal to 1:

$$
\vec{d}(\rho+1)=-T \vec{d}(\rho), \quad \rho \in \mathbb{N}
$$

Therefore,

$$
\vec{d}(\rho)=(-1)^{\rho} T^{\rho}(0, \ldots, 0,1)^{T}, \quad \rho \in \mathbb{N} .
$$

Applying the Riesz calculus for evaluating $T^{\rho}$, one obtains the following solution:

$$
d_{k}(\rho)=(-1)^{\rho}\left(\begin{array}{c}
n-k+\rho-1 \\
n-k
\end{array}\right), \quad k=0,1, \ldots, n ; n \in \mathbb{Z}_{+}, \rho \in \mathbb{N} .
$$

We shall omit the details of calculating the resolvent $(T-\lambda E)^{-1}$. We only notice that, it was convenient to subtract the subsequent rows when solving the linear system of equations $(T-$ $\lambda E) f=(0, \ldots, 0,1)^{T}$. It can be directly verified that the resulting expression (2.26) obeys (2.23), by using the Pascal identity and the induction argument.

Thus, we have obtained the following representation for $y_{n}$ :

$$
y_{n}(\rho ; x)=(-1)^{\rho} \sum_{j=0}^{n}\left(\begin{array}{c}
n-j+\rho-1 \\
n-j
\end{array}\right) \frac{x^{j}}{j !}, \quad n \in \mathbb{Z}_{+}, \rho \in \mathbb{N} .
$$

Theorem 2.1. Let $y_{n}(\rho ; x)$ be polynomials given by relation (2.27) ( $\left.\rho \in \mathbb{N}, n \in \mathbb{Z}_{+}\right)$. They have the following properties: 
(a) Polynomials $y_{n}(\rho ; x)$ admit the following representation:

$$
y_{n}(\rho ; x)=\frac{(-1)^{\rho}}{n !} x^{n}{ }_{2} F_{0}\left(-n, \rho ;-;-\frac{1}{x}\right), \quad n \in \mathbb{Z}_{+}, \rho \in \mathbb{N} ; x \in \mathbb{C} \backslash\{0\} .
$$

(b) Polynomials $y(x)=y_{n}(\rho ; x)$ satisfy the following differential equation:

$$
x \sum_{k=0}^{\rho}(-1)^{\rho-k}\left(\begin{array}{l}
\rho \\
k
\end{array}\right) y^{(k+1)}(x)-n \sum_{k=0}^{\rho}(-1)^{\rho-k}\left(\begin{array}{l}
\rho \\
k
\end{array}\right) y^{(k)}(x)=0 .
$$

(c) Polynomials $y(x)=y_{n}(\rho ; x)$ obey the following differential equation:

$$
x y^{\prime \prime}(x)-(x+\rho-1) y^{\prime}(x)-n\left[y^{\prime}(x)-y(x)\right]=0 .
$$

(d) Polynomials $y_{n}(x)=y_{n}(\rho ; x)$ are Sobolev orthogonal polynomials on $\mathbb{T}$ :

$$
\int_{\mathbb{T}}\left(y_{n}(z), y_{n}^{\prime}(z), \ldots, y_{n}^{(\rho)}(z)\right) M \overline{\left(\begin{array}{c}
y_{m}(z) \\
y_{m}^{\prime}(z) \\
\vdots \\
y_{m}^{(\rho)}(z)
\end{array}\right)} d \mu_{0}=\delta_{n, m}, \quad n, m \in \mathbb{Z}_{+},
$$

where $M$ is given by (1.5).

Proof. (a): It is readily checked that the reversed polynomial for $y_{n}$ is given by

$$
y_{n}^{*}(\rho ; x)=\frac{(-1)^{\rho}}{n !}{ }_{2} F_{0}(-n, \rho ;-;-x), \quad n \in \mathbb{Z}_{+}, \rho \in \mathbb{N},
$$

and relation (2.28) follows.

(b): Substitute for $x^{n}$ from (2.12) into the following equality:

$$
x\left(x^{n}\right)^{\prime}=n x^{n} .
$$

(c): Hypergeometric polynomials

$$
u=u_{n}(z):={ }_{2} F_{0}(-n, \rho ;-; z), \quad n \in \mathbb{Z}_{+}, \rho \in \mathbb{N},
$$

satisfy the following differential equation:

$$
z(-n+\theta)(\rho+\theta) u-\theta u=0,
$$

where $\theta=z \frac{d}{d z}$. The differential equation for the generalized hypergeometric function ${ }_{p} F_{q}$ is usually written when $p, q \geq 1$. However, the arguments in [14, p. 75] can be applied in the case $q=0$ as well. Then for $z \neq 0$, we may write

$$
z^{2} u^{\prime \prime}(z)+(\rho+1) z u^{\prime}(z)-n\left(z u^{\prime}(z)+\rho u(z)\right)-u^{\prime}(z)=0 .
$$

Observe that

$$
u_{n}(z)=\frac{n !}{(-1)^{n+\rho}} z^{n} y_{n}\left(\rho ;-\frac{1}{z}\right) .
$$

Calculating the derivatives $u_{n}^{\prime}, u_{n}^{\prime \prime}$ and inserting them into relation (2.34), after some algebraic simplifications, we get relation (2.30).

$(d)$ : This follows from our motivation and relation (1.4) in the Introduction. 
Corollary 2.1. Let $\mu$ be a probability measure on $\mathbb{T}$ with an infinite support. Denote by $p_{n}$ orthogonal polynomials on $\mathbb{T}$ with respect to $\mu$ (the positivity of leading coefficients is not assumed) which satisfy (1.1). Let

$$
p_{n}(x)=\sum_{j=0}^{n} \xi_{n, j} x^{j}, \quad \xi_{n, j} \in \mathbb{C}, \xi_{n, n} \neq 0 ; n \in \mathbb{Z}_{+} .
$$

Polynomials

$$
\widehat{y}_{n}(\rho ; x)=\sum_{j=0}^{n} \xi_{n, j} \frac{(-1)^{\rho}}{j !} x^{j}{ }_{2} F_{0}\left(-j, \rho ;-;-\frac{1}{x}\right), \quad n \in \mathbb{Z}_{+},
$$

are solutions to equation (1.3). Therefore, $\left\{\widehat{y}_{n}(\rho ; x)\right\}_{n=0}^{\infty}$ are Sobolev orthogonal polynomials satisfying relation (1.4).

Proof. Since

$$
D:=\sum_{k=0}^{\rho}(-1)^{\rho-k}\left(\begin{array}{l}
\rho \\
k
\end{array}\right) \frac{d^{k}}{d x^{k}}
$$

is a linear operator on polynomials, then we may write:

$$
\begin{gathered}
D \sum_{j=0}^{n} \xi_{n, j} \frac{(-1)^{\rho}}{j !} x^{j}{ }_{2} F_{0}\left(-j, \rho ;-;-\frac{1}{x}\right)=D \sum_{j=0}^{n} \xi_{n, j} y_{j}(\rho ; x) \\
=\sum_{j=0}^{n} \xi_{n, j} D y_{j}(\rho ; x)=\sum_{j=0}^{n} \xi_{n, j} x^{j}=p_{n}(x) .
\end{gathered}
$$

Therefore, Condition $\mathrm{A}$ is satisfied and we conclude that $\widehat{y}_{n}$ are Sobolev orthogonal polynomials.

Corollary 2.1 shows that one can take a system of OPUC with an explicit representation and construct a system of SOPUC by (2.37). For example, one can use the circular Jacobi orthogonal polynomials, see Example 8.2.5 and formulas (8.2.21), (8.2.22) in [6, pp. 229-230].

In order to obtain a recurrence relation for polynomials $y_{n}(\rho ; x)$, we shall apply Fasenmeier's method ([14]) to hypergeometric polynomials $u_{n}(z)$ from (2.32). In the following considerations, we shall admit for $\rho$ to be not only positive integer values but $\rho>0$ as well. We shall express $u_{n}, u_{n-1}, u_{n-2}, z u_{n}(z), z u_{n-1}(z)$, using $u_{n+1}(z)$. Choose and fix an arbitrary integer $n$ greater than or equal to 2 . We may write

$$
u_{n+1}(z)=\sum_{k=0}^{\infty}(-n-1)_{k}(\rho)_{k} \frac{z^{k}}{k !}=\sum_{k=0}^{\infty} \varepsilon_{n+1}(k),
$$

where $\varepsilon_{n+1}(k)=\varepsilon_{n+1}(z ; \rho ; k):=(-n-1)_{k}(\rho)_{k} \frac{z^{k}}{k !}$. Using

$$
\begin{gathered}
(-n)_{k}=(-n-1)_{k} \frac{(n+1-k)}{(n+1)}, \quad k \in \mathbb{Z}_{+}, \\
(-n+1)_{k}=(-n-1)_{k} \frac{(n+1-k)(n-k)}{(n+1) n}, \quad k \in \mathbb{Z}_{+},
\end{gathered}
$$

and similar relations, we obtain that

$$
u_{n}(z)=\sum_{k=0}^{\infty} \varepsilon_{n+1}(k) \frac{(n+1-k)}{(n+1)}
$$




$$
\begin{gathered}
u_{n-1}(z)=\sum_{k=0}^{\infty} \varepsilon_{n+1}(k) \frac{(n+1-k)(n-k)}{(n+1) n}, \\
u_{n-2}(z)=\sum_{k=0}^{\infty} \varepsilon_{n+1}(k) \frac{(n+1-k)(n-k)(n-1-k)}{(n+1) n(n-1)}, \\
z u_{n}(z)=\sum_{k=0}^{\infty} \varepsilon_{n+1}(k) \frac{(-k)}{(n+1)(\rho+k-1)}, \quad \rho \neq 1, \\
z u_{n-1}(z)=\sum_{k=0}^{\infty} \varepsilon_{n+1}(k) \frac{(-k)(n+1-k)}{n(n+1)(\rho+k-1)}, \quad \rho \neq 1 .
\end{gathered}
$$

We now assume that $\rho \neq 1$. Consider the following expression $R_{n}(z)$ :

$$
\begin{aligned}
R_{n}(z):= & \varphi_{1} u_{n-1}(z)+\varphi_{2} u_{n}(z)+\varphi_{3} u_{n+1}(z)+\varphi_{4} z u_{n}(z)+ \\
& +\varphi_{5} z u_{n-1}(z)+\varphi_{6} u_{n-2}(z), \quad \varphi_{k} \in \mathbb{C} .
\end{aligned}
$$

We intend to choose parameters $\varphi_{k}$ (depending on the chosen $n$ ) in such a way that $R_{n}(z)=0$, $\forall z \in \mathbb{C}$. Substitute above expressions for $u_{n-2}, u_{n-1}, u_{n}, z u_{n}, z u_{n-1}$ into (2.43) to get

$$
R_{n}(z)=\sum_{k=0}^{\infty} \varepsilon_{n+1}(k) \frac{1}{(n-1) n(n+1)(\rho+k-1)} I_{n, k}
$$

where

$$
\begin{aligned}
& I_{n, k}=\varphi_{1}(n-k)(n+1-k)(n-1)(\rho+k-1)+\varphi_{2}(n+1-k)(n-1) n(\rho+k-1)+ \\
& +\varphi_{3}(n-1) n(n+1)(\rho+k-1)+\varphi_{4}(-1) k(n-1) n+\varphi_{5}(n+1-k)(-1) k(n-1)+ \\
& \quad+\varphi_{6}(n+1-k)(n-k)(n-1-k)(\rho+k-1) .
\end{aligned}
$$

Observe that $I_{n, k}$ is a polynomial of degree $\leq 4$. Therefore, we may check that $I_{n, k}=0$ for some distinct five values of $k$ to get $R_{n}(z) \equiv 0$. This is a crucial point in the Fasenmeier's method.

We choose $k=-\rho+1 ; n+1 ; n ; n-1 ; 0$. After some obvious simplifications, we get the following five equations:

$$
\begin{gathered}
\varphi_{5}=-\frac{n \varphi_{4}}{n+\rho}, \\
\varphi_{3}=\frac{\varphi_{4}}{n+\rho}, \\
\varphi_{2}(\rho+n-1)+\varphi_{3}(n+1)(\rho+n-1)+\varphi_{4}(-1) n-\varphi_{5}=0, \\
\varphi_{1} 2(\rho+n-2)+\varphi_{2} 2 n(\rho+n-2)+\varphi_{3} n(n+1)(\rho+n-2)- \\
-\varphi_{4}(n-1) n-\varphi_{5} 2(n-1)=0, \\
\varphi_{1}+\varphi_{2}+\varphi_{3}+\varphi_{6}=0 .
\end{gathered}
$$

Set $\varphi_{4}=n+\rho$. Then,

By (2.47), we get

$$
\varphi_{5}=-n, \quad \varphi_{3}=1 .
$$

$$
\varphi_{2}=-1 \text {. }
$$


By (2.48), we obtain that $\varphi_{1}=0$. Finally, by (2.49) we conclude that $\varphi_{6}=0$, as well. Consequently, polynomials $u_{n}(z)$ satisfy the following relation:

$$
\begin{gathered}
u_{n+1}(z)-u_{n}(z)+(n+\rho) z u_{n}(z)-n z u_{n-1}(z)=0, \\
n=2,3, \ldots ; \rho>0, \rho \neq 1 .
\end{gathered}
$$

It is directly checked that, relation (2.50) holds for the values $n=0,1$, if we set $u_{-1}:=0$. Moreover, we can take limit when $\rho \rightarrow 1$, to prove that relation (2.50) holds for $\rho=1 ; n \in \mathbb{Z}_{+}$:

$$
u_{n+1}(z)-u_{n}(z)+(n+\rho) z u_{n}(z)-n z u_{n-1}(z)=0, \quad n \in \mathbb{Z}_{+} ; \rho>0 .
$$

Theorem 2.2. Let $y_{n}(x)=y_{n}(\rho ; x)$ be polynomials from relation (2.27) with $\rho \in \mathbb{N}$. They satisfy the following recurrence relation:

$$
(n+1) y_{n+1}(\rho ; x)-(n+\rho) y_{n}(\rho ; x)=x\left(y_{n}(\rho ; x)-y_{n-1}(\rho ; x)\right), \quad n \in \mathbb{Z}_{+},
$$

where $y_{-1}(\rho ; x):=0$

Proof. Use relations (2.35) and (2.50).

Relation (2.52) can be written in the following matrix form:

$$
\widehat{L} \vec{y}(\rho ; x)=x \widehat{M} \vec{y}(\rho ; x),
$$

where $\vec{y}(\rho ; x)=\left(y_{0}(\rho ; x), y_{1}(\rho ; x), \ldots\right)^{T}$. The semi-infinite matrix $\widehat{M}$ is two-diagonal, having all 1 on the main diagonal, and all -1 on the first sub-diagonal. The semi-infinite matrix $\widehat{L}$ is also two-diagonal, but having $(-\rho,-\rho-1,-\rho-2, \ldots)$ on the main diagonal, and $(1,2,3, \ldots)$ on the first upper diagonal.

Denote by $\widehat{L}_{n}\left(\widehat{M}_{n}\right)$ the $(n+1) \times(n+1)$ matrix standing on the intersection of the first $(n+1)$ rows and the first $(n+1)$ columns of $\widehat{L}$ (respectively $\widehat{M})$. Let $x^{*}$ be an arbitrary root of $y_{n+1}(\rho ; x)$. Then

$$
\widehat{L}_{n} \vec{y}\left(\rho ; x^{*} ; n\right)=x^{*} \widehat{M} \vec{y}\left(\rho ; x^{*} ; n\right),
$$

where $\vec{y}\left(\rho ; x^{*} ; n\right)=\left(y_{0}\left(\rho ; x^{*}\right), y_{1}\left(\rho ; x^{*}\right), \ldots, y_{n}\left(\rho ; x^{*}\right)\right)^{T}$. Thus, there is a link between the analytic theory of polynomials (the location of zeros) and the matrix theory (generalized eigenvalue problems, see [5]).

Notice that, monic polynomials $\widetilde{y}_{n}(\rho ; x)$ are given by

$$
\widetilde{y}_{n}(\rho ; x)=\frac{n !}{(-1)^{\rho}} y_{n}(\rho ; x), \quad n \in \mathbb{Z}_{+}, \rho \in \mathbb{N} .
$$

The recurrence relation (2.52) takes the following form (we shifted the indices):

$$
\widetilde{y}_{n}(\rho ; x)=(x+n-1+\rho) \widetilde{y}_{n-1}(\rho ; x)-(n-1) x \widetilde{y}_{n-2}(\rho ; x), \quad n \in \mathbb{N} .
$$

Relation (2.55) is a particular case of a general recurrence relation (2.1) on page 5 in [7]. This general recurrence relation is related to $R_{I}$-fractions and biorthogonal rational functions [7, Theorem 2.1].

Finally, observe that $y_{1}(\rho ; x)=(-1)^{\rho}(x+\rho)$. Thus, $y_{1}(1 ; x)$ has its root on the unit circle, while the roots of $y_{1}(\rho ; x)$, for $\rho>1$, are outside $\mathbb{T}$. Consequently, polynomials $y_{n}(\rho ; x)$ are not orthogonal on the unit circle with respect to a scalar measure. 


\section{REFERENCES}

[1] L. C. Andrews: Special functions of mathematics for engineers. Reprint of the 1992 second edition. SPIE Optical Engineering Press, Bellingham, WA; Oxford University Press, Oxford, (1998).

[2] H. Azad, A. Laradji and M. T. Mustafa: Polynomial solutions of differential equations. Adv. Difference Equ. 2011:58 (2011), $12 \mathrm{pp}$.

[3] K. Castillo: A new approach to relative asymptotic behavior for discrete Sobolev-type orthogonal polynomials on the unit circle. Appl. Math. Lett. 25 (2012), no. 6, 1000-1004.

[4] L. Garza, F. Marcellán and N. C. Pinzón-Cortés: (1,1)-coherent pairs on the unit circle. Abstr. Appl. Anal. (2013), Art. ID 307974, 8 pp.

[5] Kh. D. Ikramov: Matrix pencils - theory, applications, numerical methods. (Russian) Translated in J. Soviet Math. 64 (1993), no. 2, 783-853. Itogi Nauki i Tekhniki, Mat. Anal., 29, Mathematical analysis, Vol. 29 (Russian), 3-106, Akad. Nauk SSSR, Vsesoyuz. Inst. Nauchn. i Tekhn. Inform., Moscow, (1991).

[6] M. E. H. Ismail: Classical and quantum orthogonal polynomials in one variable. With two chapters by Walter Van Assche. With a foreword by Richard A. Askey. Encyclopedia of Mathematics and its Applications, 98. Cambridge University Press, Cambridge, (2005).

[7] M. E. H. Ismail, D. R. Masson: Generalized orthogonality and continued fractions. J. Approx. Theory 83 (1995), no. 1, $1-40$.

[8] K. H. Kim, H. K. Kwon, L. L. Littlejohn and G. J. Yoon: Diagonalizability and symmetrizability of Sobolev-type bilinear forms: a combinatorial approach. Linear Algebra Appl. 460 (2014), 111-124.

[9] R. Koekoek, P. A. Lesky and R. F. Swarttouw: Hypergeometric orthogonal polynomials and their q-analogues. With a foreword by Tom H. Koornwinder. Springer Monographs in Mathematics. Springer-Verlag, Berlin, (2010).

[10] K. H. Kwon, L. L. Littlejohn and G. J. Yoon: Ghost matrices and a characterization of symmetric Sobolev bilinear forms. Linear Algebra Appl. 431 (2009), no. 1-2, 104-119.

[11] F. Marcellán, Y. Xu: On Sobolev orthogonal polynomials. Expo. Math. 33 (2015), no. 3, 308-352.

[12] A. S. Markus: Introduction to the spectral theory of polynomial operator pencils. With an appendix by M. V. Keldysh. Translations of Mathematical Monographs, 71. American Mathematical Society, Providence, RI, (1988).

[13] R. Mennicken, M. Möller: Non-self-adjoint boundary eigenvalue problems. North-Holland Mathematics Studies, 192. North-Holland Publishing Co., Amsterdam, (2003).

[14] E. D. Rainville: Special functions. Reprint of 1960 first edition. Chelsea Publishing Co., Bronx, N.Y., (1971).

[15] L. Rodman: An introduction to operator polynomials. Operator Theory: Advances and Applications, 38. Birkhäuser Verlag, Basel, (1989).

[16] B. Simon: Orthogonal polynomials on the unit circle. Part 1. Classical theory. American Mathematical Society Colloquium Publications, 54, Part 1. American Mathematical Society, Providence, RI, (2005).

[17] B. Simon: Orthogonal polynomials on the unit circle. Part 2. Spectral theory. American Mathematical Society Colloquium Publications, 54, Part 2. American Mathematical Society, Providence, RI, (2005).

[18] A. Sri Ranga: Orthogonal polynomials with respect to a family of Sobolev inner products on the unit circle. Proc. Amer. Math. Soc. 144 (2016), no. 3, 1129-1143.

[19] G. Szegö: Orthogonal polynomials. Fourth edition. American Mathematical Society, Colloquium Publications, Vol. XXIII. American Mathematical Society, Providence, R.I., (1975).

[20] S. M. Zagorodnyuk: On some classical type Sobolev orthogonal polynomials. J. Approx. Theory 250 (2020), 105337, 14 pp.

[21] A. Zhedanov: Biorthogonal rational functions and the generalized eigenvalue problem. J. Approx. Theory 101 (1999), no. 2, pp. 303-329.

V. N. KARAZIN KHARKIV NATIONAL UNIVERSITY

SCHOOL OF MATHEMATICS AND COMPUTER SCIENCES

SVOBODY SQUARE 4, 61022, KHARKIV, UKRAINE

ORCID: 0000-0002-1063-1776

E-mail address: Sergey.M. Zagorodnyuk@gmail.com 